\title{
Functional brain and age-related changes associated with congruency in task switching
}

\author{
Teal S. Eich*, David Parker, Dan Liu, Hwamee Oh, Qolamreza Razlighi, Yunglin Gazes, \\ Christian Habeck, Yaakov Stern
}

Cognitive Neuroscience Division and The Taub Institute, Department of Neurology, Columbia University Medical Center, United States

\section{A R T I C L E I N F O}

\section{Article history:}

Received 10 March 2016

Received in revised form

8 August 2016

Accepted 9 August 2016

Available online 9 August 2016

\section{Keywords:}

Cognitive control

Aging

Task switching

Congruency

Response conflict

fMRI

\begin{abstract}
A B S T R A C T
Alternating between completing two simple tasks, as opposed to completing only one task, has been shown to produce costs to performance and changes to neural patterns of activity, effects which are augmented in old age. Cognitive conflict may arise from factors other than switching tasks, however. Sensorimotor congruency (whether stimulus-response mappings are the same or different for the two tasks) has been shown to behaviorally moderate switch costs in older, but not younger adults. In the current study, we used fMRI to investigate the neurobiological mechanisms of response-conflict congruency effects within a task switching paradigm in older $(N=75)$ and younger $(N=62)$ adults. Behaviorally, incongruency moderated age-related differences in switch costs. Neurally, switch costs were associated with greater activation in the dorsal attention network for older relative to younger adults. We also found that older adults recruited an additional set of brain areas in the ventral attention network to a greater extent than did younger adults to resolve congruency-related response-conflict. These results suggest both a network and an age-based dissociation between congruency and switch costs in task switching.
\end{abstract}

c 2016 Elsevier Ltd. All rights reserved.

\section{Introduction}

The ability to flexibly control cognitive systems is critical for adaptive human behavior. Yet, the systems that underlie these abilities are far from being perfectly efficient, even under optimal circumstances. A case in point is the ubiquitous finding within the task switching literature of poorer performance when participants must alternate between completing two simple tasks versus completing either task alone (Gopher et al., 2000; Jersild, 1927; Kramer et al., 1999; Rogers and Monsell, 1995; and see Kiesel et al. (2010), for a recent review). A distributed network of prefrontal, fronto-parietal and striatal regions has been associated with the increased demands of switching versus repeating tasks (Badre and Wagner, 2006; Brass and von Cramon, 2002; Braver et al., 2003; Reynolds and O'Reilly, 2009).

Changes to functional activation in aging, including reduced hemispheric lateralization and engagement of supplementary brain areas, are particularly salient in frontally-mediated tasks that require executive control (Cabeza, 2002). Given this, it is not surprising that task switching effects have been shown to increase in

\footnotetext{
* Correspondence to: Cognitive Neuroscience Division, Department of Neurology and The Taub Institute, Columbia University, 630 W. 168th St., P \& S Box 16, New York, NY 10032, United States.

E-mail address: tse4@columbia.edu (T.S. Eich).
}

older as compared to younger adults (Botwinick et al., 1958; Hartley et al., 1990; Kramer et al., 1999; Kray and Lindenberger, 2000; Verhaeghen, 2011; Wasylyshyn et al., 2011), and that these age-related changes to behavioral performance are associated with changes in neural patterns of activation, including under-recruitment (deactivations and a decreased spatial extent of activation in the fronto-parietal network), a shift from unilateral to bilateral activations, and the recruitment of additional brain areas (DiGirolamo et al., 2001; Milham et al., 2002). Whereas under-recruitment has been associated with older adults' decreased behavioral performance relative to younger adults, a compensatory mechanism is often put forth to explain the activation of bilateral and additional brain areas in the older adults (Cabeza, 2002; Cabeza et al., 2002).

Mediating between competing tasks with different decision rules is not the only source of cognitive conflict within a typical task switching paradigm, however. When bivalent stimuli are used, response overlap across task sets can occur, and a single stimulus can produce conflicting responses for the two task dimensions (Braver et al., 2003; Derrfuss et al., 2004). This type of congruency-related conflict was interrogated by Rogers and Monsell in their seminal task switching paradigm (1995). In their task, the location within a $2 \times 2$ quadrant served as a cue for which of two decision rules should be adopted about letter-number stimulus pairs. When the pair appeared in one of the top 2 positions, 
participants made a vowel/consonant decision about the letter, pressing left for vowel and right for consonant. When it appeared in one of the bottom 2 positions, participants made a parity decision about the number, pressing left for odd and right for even. The responses to the stimulus "A3" is "congruent", since participants should press the same button regardless of where in the grid the stimulus appeared. However, for the stimuli pair 'A2', 'A' requires a left button press, while ' 2 ' calls for a right hand button press. In these types of trials, the participant must apply the appropriate decision rule, based on the location of the stimulus in the grid, to get the trial correct. This type of stimulus is referred to as "incongruent" since the two decisions rules call for opposing responses. In these cases, cognitive control is necessary to overcome stimulus-response-conflict, which relates to the sensorimotor mapping of the decision rule for each stimulus to a limited number of possible response options (Fitts and Deininger, 1954; Fitts and Seeger, 1953). According to the dimensional overlap model (Kornblum and Lee, 1995), resolving this type of responseconflict demands a complex set of response-selection processes that may include inhibiting irrelevant stimulus-response associations and employing working memory and response-selection processes to establish the correct response from the active set of stimulus response pairings.

Studies investigating the behavioral correlates of responseconflict congruency effects on task switching performance in younger adults have found that congruent trials produced quicker and more accurate responses do than incongruent trials (Sudevan and Taylor, 1987), an effect that Meiran (2005) termed the Task Rule Congruency Effect. Fagot (1994) reported that congruency did not alter local switch costs (when performance on switch and noswitch trials within a dual-task block are compared (e.g. Kray and Lindenberger, 2000; Mayr, 2001; Verhaeghen and Cerella, 2002)), but did affect local mixing costs (the change in performance on no-switch trials in dual-task blocks compared to no-switch trials in single-task blocks). Meiran and colleagues (Meiran, 2000a,b; Meiran and Kessler, 2008) similarly reported reaction time increases for incongruent relative to congruent trials in younger adults, and proposed that these increased latencies reflect the additional time needed to resolve interference resulting from response category codes activated in long term memory.

The effects of age on task switching response-conflict congruency have only been investigated in two published studies that we are aware of Meiran et al. (2001) found a significant three-way interaction between task switching, congruency and age, such that there was "a larger congruency effect in the switch condition relative to the no-switch condition" (p. 93) for the older adults relative to the younger adults in at least one of their experiments. Our group has also previously reported interactions between congruency and task switching, such that the response-selection process underlying the congruency manipulation was found to moderate the decrements to performance associated with going from single to dual-task blocks in older adults, but not in younger adults (Eich et al., 2015). Taken together, these findings suggest that the typically seen age-related increases in switch costs may be derivative upon a more basic response-conflict congruency effect.

The neural basis of response-conflict congruency effects in the context of task switching, in either younger or older adults, remains elusive. Congruency effects have, however, been studied extensively in paradigms like the Stroop (Stroop, 1935; and see MacLeod (1991) for a review), Simon (Simon and Rudell, 1967), and Flanker (Eriksen and Eriksen, 1974) tasks. These tasks have uncovered discrete neural networks, including the dorsal anterior cingulate cortex (ACC) and the dorsolateral prefrontal cortex (DLPFC) associated with processing incongruent information (Botvinick et al., 2001; Durston et al., 2003; Fan et al., 2003; Milham and Banich, 2005; Milham et al., 2003; Ullsperger and von
Cramon, 2003; vanVeen and Carter, 2005). These brain areas thought to represent the neural correlates of remedial cognitive control processes serving to detect and resolve response-conflict by suppressing the responses that are automatically triggered by attributes of the presented stimulus in favor of goal-relevant responses (Kane and Engle, 2003).

Derrfuss et al. (2004) showed that a specific region in the posterior frontolateral cortex, the Inferior Frontal Junction (IFJ), was bilaterally and commonly activated in a task switching paradigm, the Stroop task, and a verbal N-back task. Although congruency was not directly explored in their task switching paradigm, the stimuli were bivalent, and thus sensorimotor responseconflict congruency was operative: participants indicated whether a number-stimulus was lower than 5 or even using their index finger, or 5 or higher or odd with their middle finger. A subsequent meta-analysis revealed significant IFJ activations across tasks that involve task switching and Stroop paradigms (Derrfuss et al., 2005). These results suggest that switch costs and response-conflict related congruency effects may share a common neurobiological mechanism.

However, there is debate as to whether the congruency effects seen in discrimination tasks like the Stroop, Simon and Flanker, that emphasize fast responding, are equivalent in mechanism to task switching congruency effects. Meiran (2005) found that spatial congruency effects in the Simon task were additive with task switching congruency effects. Meiran and Kessler (2008) argued that task switching congruency effects are fundamentally different and independent from Stroop effects, as the category-response mappings in task switching experiments are arbitrary and novel, existing only within the context of the experiment, whereas those in the Stroop task are direct, implicit and pre-existing, having been learned over the course of a lifetime. They provide evidence for a dissociation between the congruency processes that are active in task switching and Stroop paradigms, showing that task switching congruency effects arise when a novel stimulus-response association is learned, but do not occur even after substantial training of stimulus-response mappings if these mappings are direct and do not involve a categorical intermediate.

In the current study, we set out to investigate whether the neurobiological mechanisms involved in switch costs and response-conflict congruency effects overlap, or if instead are dissociable. It is possible that there might be an age-related dissociation between these types of cognitive processes. This possibility would echo the results of found in our behavioral data using a similar task switching paradigm with a congruency manipulation (Eich et al., 2015). In the imaging data, the younger adults may recruit a common set of areas to resolve both switching and congruency related conflicts, but the added cognitive load that both switching between different tasks and resolving stimulusresponse incongruency levies on older adults may result in older adults activating different brain areas; either a more extensive network, or different areas altogether. This possibility is strengthened by robust age-related changes to neural circuitry. To investigate these possibilities, we used functional magnetic resonance imaging (fMRI) to compare blood oxygen level-dependent activity in a large, well characterized, education matched sample of 20-30 year olds, and clinically healthy non-demented 60-70 year olds. Participants performed an fMRI-optimized version of our behavioral task switching paradigm that allowed us to separate task switching effects (dual-task versus single-task performance) from congruency effects. 


\section{Materials and methods}

\subsection{Participants}

A total of 150 participants completed the study. Data from 13 participants (4 younger, 9 older) were excluded for having below chance performance on incongruent trials (less than $50 \%$ accuracy, $N=3$ ), or for having not responded on more than one quarter of trials $(N=10)$. Our final sample consisted of 137 participants. Sixty-two were younger adults (age range $20-30, M$ age $=25.82,62.9 \%$ female, $M$ years education=15.57) and seventy-five were older adults (age range 60-70, $M$ age $=64.84,56 \%$ female, $M$ years education $=15.99$ ). Education did not differ between older and younger adults $(t(135)=-1.02, p>.25)$. All participants were screened for current neurological or psychiatric diagnoses, and medication use. Older adults were further screened for dementia via the Dementia Rating Scale (Mattis, 1988), and any participant with a score above 135 was not eligible to participate. Informed consent, as approved by the Internal Review Board of the College of Physicians and Surgeons of Columbia University, was obtained prior to study participation for all participants.

\subsection{Stimuli}

The stimuli consisted of 12 letter-stimuli, presented in red, green or white, pseudo-randomly chosen from the set (A, E, I, O, a, e, i, o, C, G, K, P, c, g, k, p). The task stimuli were back-projected onto a black screen located at the foot of the MRI bed using an LCD projector. Participants viewed the screen via a mirror system located in the head coil and, if needed, had vision corrected to normal using MR compatible glasses (manufactured by SafeVision, LLC. Webster Groves, MO).

\subsection{Experimental protocol}

The experimental procedure was modeled after Experiment 2 of Koechlin et al. (2003), an intrinsically cued task switching paradigm with a no-go component. Participants were presented with bivalent letter stimuli, where the color of the letter served as an intrinsic cue for which type of decision should be made. If the letter was red, participants made an upper-case/lower-case decision, using the left index finger to indicate a lower-cased letter and the right index finger for upper-cased letters. If the letter was green, participants decided if it was a vowel or a consonant, pressing with the left index finger for vowels and the right index finger for consonants. If the letter was white, participants withheld a response (no-go trials). Switch costs were calculated as a function of the number of tasks that the participant had to complete within a block of trials. In single-task blocks, participants made only vowel/consonant or upper/lower case decisions. In dual-task blocks, participants had to switch between performing vowel/ consonant and upper/lower case decisions, based on the color of the letter. Stochastic switching, as opposed to patterned switching, was employed so that from trial-to-trial the expectancy of a switch between tasks was reduced (Monsell et al., 2003).

Congruency was manipulated on a trial-by-trial basis. Every stimulus was either an upper or lower-cased vowel or consonant. Thus, each stimulus potentially informed a response for both tasks; only the color of the stimulus determined which response should be made on each trial. A single stimulus could therefore indicate either congruent (when the response for both tasks required the same key-press; i.e., lower-cased vowels or upper-case consonants) or incongruent (when the response for one task is in opposition to the required response for the other task; i.e., uppercase vowels and lower-case consonants) responses for the two stimulus dimensions. Because of this, the task design meets the conditions for dimensional overlap (Kornblum and Lee, 1995) necessary to create stimulus-response incongruency (Fitts and Deininger, 1954; Fitts and Seeger, 1953) and the drive for responseselection demands (Lien and Proctor, 2002; Pashler, 1994) to resolve the conflict. Response-conflict is operative in dual-task blocks when both tasks are active. But, critically, it may also be present in single-task blocks when only one task is active because both dimensions are always defined for each stimulus.

In the scanner, participants received a total of 36 blocks, broken into 6 fMRI runs. Twenty-four blocks, each $33.5 \mathrm{~s}$, included twelve $2.4 \mathrm{~s}$ trials, with an instruction cue presented for $2.8 \mathrm{~s}$, followed by a blank screen for $1.9 \mathrm{~s}$, followed by a letter-stimulus. Jitter was introduced in two ways: first, each stimulus was terminated and replaced by a blank screen when a response was made or after $2350 \mathrm{~ms}$, whichever occurred first (see Fig. 1A). Second, four no-go trials (in which the correct response was to withhold a response) were interspersed within the 8 experimental trials in each block. Participants completed four conditions, two no-switch conditions (Fig. 1B) and two identical switch conditions (Fig. 1C), a total of six times. In all blocks, stimuli were counterbalanced so that (1) no more than two task or no-go trials occurred in a row; (2) no more than two vowel/consonant or upper/lower case task trials occurred in a row in dual-task blocks; and (3), congruent and incongruent trials were equally likely and repeated on no more than two successive trials. The remaining 12 blocks consisted of resting conditions (also $33.5 \mathrm{~s}$ in duration each), in which no stimuli were presented and no response was required. Each resting block presented an instruction cue ("REST") for $2.8 \mathrm{~s}$, followed by a blank screen for the remainder of the run. Rest blocks were not modeled, and were included in the experimental paradigm for analyses unrelated to the current study. Before beginning the experiment, outside of the scanner, participants completed a three-part training session. Participants were first pre-trained on the task, then they received between one and three blocks of each condition with auditory feedback indicating incorrect responses, and finally they were tested on the entire paradigm without feedback. In the scanner, responses were made on a LUMItouch response system (Photon Control Company) using the left and right index fingers. Feedback was not given. Task administration and collection of behavioral data were controlled using PsyScope 5X B53 (Macwhinney et al., 1997) running on a Macintosh G3/G4 iBook. Digital input-output for the response system and synchronization with the MRI acquisition computer, as well as millisecond accurate timing of responses, was provided by a MellonIOLabs Systems USB Button Box.

\subsection{MRI data acquisition}

Multislice images of the human brain were acquired in an event-related design using a 3.0 T Philips Achieva Magnet equipped with standard quadrature head coil.

\subsection{Data analysis}

\subsubsection{Behavioral data}

The data were analyzed using SPSS 22. For all participants, trials on which a response was made (correct or incorrect) in less than 300 ms were excluded. Following Eich et al. (2015), our primary behavioral variable of interest was accuracy (percent correct). Mixed-models repeated measures ANOVA and $t$-tests were used to examine the cross sectional effects of Task (single versus dual) and Congruency (congruent versus incongruent) on Age (young versus old).

\subsubsection{Anatomical MRI data}

A T1-weighted scout image was acquired to determine 

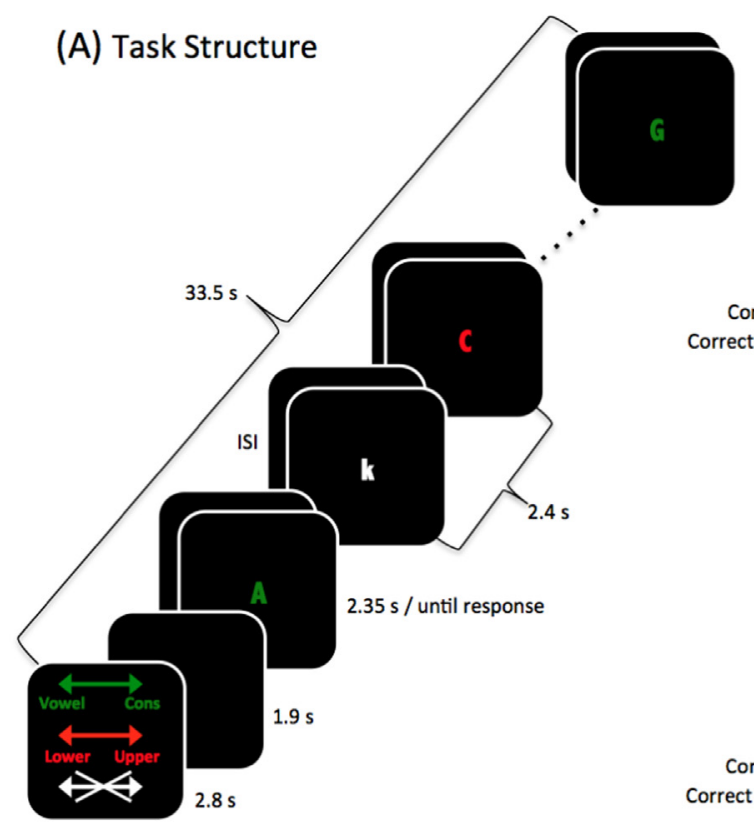

(B) Single-Task
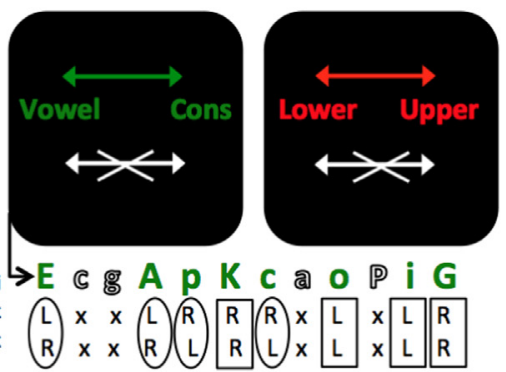

(C) Dual-Task
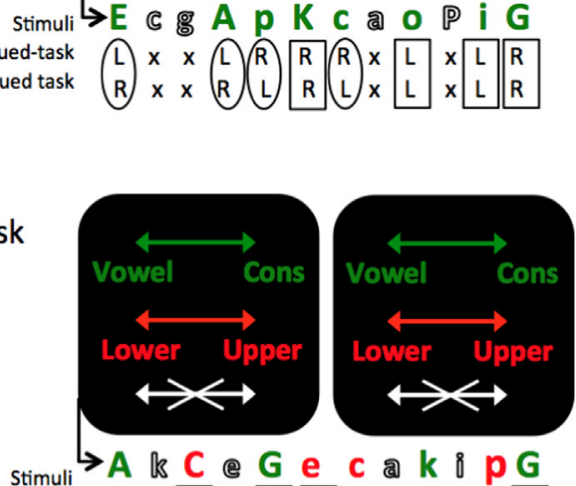

Stimuli

Correct response for cued-task

Correct response for non-cued task

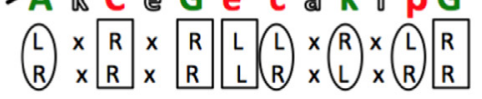

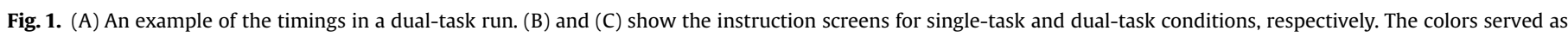

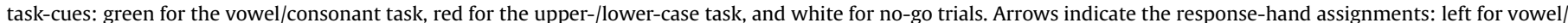

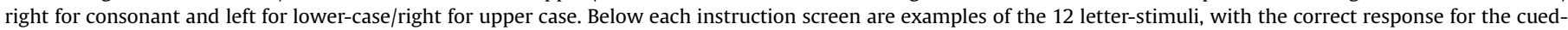

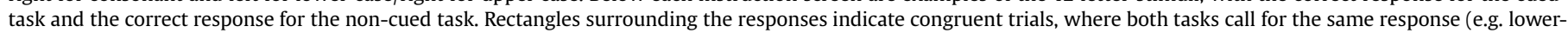

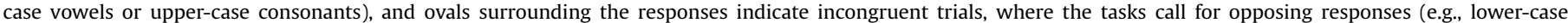
consonants or upper-case vowels).

participant position. One hundred and sixty five contiguous $1 \mathrm{~mm}$ axial T1-weighted images of the whole brain were acquired for each participant with an MPRAGE sequence using the following parameters: TR $6.5 \mathrm{~ms}$, TE $3 \mathrm{~ms}$; flip angle 8, in-plane acquisition matrix $256 \times 256$ and $25.6 \times 25.6 \mathrm{~cm}$ field of view which results in an isometric voxel size of $1 \times 1 \times 1 \mathrm{~mm}$. All T1 scans were reviewed by a neuroradiologist for evidence of potentially clinically significant findings such as abnormal neural structure. No clinically significant findings were identified.

\subsubsection{Functional MRI data}

Functional data were acquired on the same Philips scanner. Each run included collection of 111 functional volumes. Forty-one axial slices per volume, with a $3.0 \mathrm{~mm}$ thickness and no gap in between, were acquired using a field echo echo-planar imaging (FE-EPI) sequence with the following parameters: TR $2000 \mathrm{~ms}$, TE $20 \mathrm{~ms}$, flip angle 72; in-plane acquisition matrix $112 \times 112$ matrix; this results in a voxel size of $2.0 \times 2.0 \times 3.0 \mathrm{~mm}$. Before the initiation of the task, four volumes were acquired and discarded to allow transverse magnetization immediately after radio-frequency excitation to approach its steady-state value.

\subsection{4. fMRI pre-processing}

FMRIB Software Library v5.0 (FSL) and custom-written Python code were used to perform the following pre-processing steps for each participant's data set: all functional images were realigned to the first volume, corrected for the order of slice acquisition, smoothed with a $5 \mathrm{~mm}^{3}$ non-linear kernel followed by intensity normalization, and high-pass filtered using a Gaussian kernel and cut-off frequency of $.008 \mathrm{~Hz}$. For spatial normalization, the accompanying T1-weighted high-resolution anatomic image was coregistered to the first functional volume using the mutual information co-registration algorithm implemented in FLIRT. This co-registered high-resolution image was then registered to MNI standardized space. These obtained transformation parameters were used to transfer the statistical parametric maps of the subject level analysis to standard space.

\subsection{5. fMRI subject level analysis}

The fMRI time-series data was pre-whitened to explicitly correct for intrinsic autocorrelations in the data. The FEAT module in FSL was used for first-level analysis. An event-related design was used to model the fMRI data, allowing us to separate timeouts (where no response was made), correct and incorrect trials, as well as congruent and incongruent trials. Errors and time outs were modeled together. Rest blocks were not included in the model. For all participants, a first level analysis was run on each of their 6 task-based runs with four regressors. The regressors represented one of the following task conditions: single-task congruent trials, single-task incongruent trials, dual-task congruent trials, dual-task incongruent trials. The regressors were generated by convolving FSL's double gamma canonical HRF with the duration of presentation of the stimulus to the participants. A second level analysis was run on each participant by combining all six first level results for each run. At this level, four new contrasts were created from the combined first level task regressors for each participant: (1) dual-task trials greater than all single-task trial activations; (2) dual-task incongruent trials greater than single-task incongruent trials; (3) incongruent trial activations greater than congruent trial activations; and (4) single-task incongruent trials greater than single-task congruent trials.

\subsection{6. fMRI group level analyses}

After transforming each participant's statistical parametric maps (obtained from the second level analysis) into standard space, group level analyses were performed using General Linear Model (GLM) with FLAME in FSL. The four participant level contrasts described above were passed into the group level analysis, where the participants were divided into "young" and "old" groups based upon their age $(20-30 ; 60-70)$. The group level parametric map was then computed for each of the four contrasts, with the following group comparisons: young only, old only, young greater 
than old, and old greater than young. In total, 16 group level contrasts were generated. Voxel-wise statistical height thresholds, combined with cluster level thresholds, were employed to ensure appropriate control over false-positives. Cluster analysis, identified as voxels with a $z$ value greater than 2.3 , and that are connected to another voxel by at least a point, were performed on the group level FSL FLAME results. We used a smoothness estimate of the data to implement Gaussian Random Field theory to infer the significance of each cluster (Worsley et al., 1992). Only clusters with an inferred significance of $p<.01$ were included in the analysis, except where noted.

\section{Results}

\subsection{Behavioral data}

A repeated measures ANOVA on accuracy (percent correct) with Task (single versus dual-task) and Congruency (congruent versus incongruent trials) as within subjects factors, and Age (younger versus older adults) as a between subject factor revealed significant main effects of Task $\left(F(1,135)=90.42, p<.001, \eta_{\mathrm{p}}^{2}=.4\right)$ and Congruency $\left(F(1,135)=109.64, p<.001, \eta_{p}^{2}=.45\right)$. The main effect of Age approached significance $(F(1,135)=3.54, p=.06$, $\left.\eta_{\mathrm{p}}^{2}=.03\right)$. There were significant two-way interactions between Task $\times$ Age $\left(F=12.01, p=.001, \quad \eta_{\mathrm{p}}^{2}=.08\right)$, Congruency $\times$ Age $(F(1$, $\left.135)=5.85, p=.02, \eta_{\mathrm{p}}^{2}=.04\right)$ and Task $\times$ Congruency $(F(1,135)=$ 49.52, $\left.p<.001, \eta_{\mathrm{p}}^{2}=.27\right)$. However, the significant two-way interactions were moderated by a significant three-way interaction between Task, Congruency and Age $(F(1,135)=3.89, p=.05$, $\left.\eta_{\mathrm{p}}^{2}=.03\right)$. The Task $\times$ Congruency $\times$ Age interaction reveals that accuracy is especially impaired in older adults for incongruent trials in dual-task blocks. This was confirmed with posthoc $t$-test which showed equivalent performance for older and younger adults on single-task congruent trials $(t(135)=-.47, p>.6)$, single-task incongruent trials $(t(135)=1.13, p>.25)$, and dual-task congruent trials $(t(135)=.98, p>.3)$. However, as can be seen in Fig. 2, on the dual-task incongruent trials, older adults performed significantly more poorly than the younger adults (89\% vs. $82 \%$; $t$ $(135)=2.82, p=.006,95 \% \mathrm{CI}[.02-.019])$. Thus, the age-related performance difference in task switching stems from an interactive effect of both Task and Congruency.

In addition to this model, we also performed 2 sets of planned ANOVAs in order to isolate the effects of task switching and response-conflict congruency. For the first contrast, we compared performance on single-task incongruent trials versus dual-task incongruent trials (Pure Task) as a measure of pure task switching load. This was based on the fact that an accurate response on either of these types of incongruent trials requires a participant to must adopt the correct decision-rule, which is not the case for congruent trials, where a participant might adopt the wrong rule, but still get the trial correct because both responses are accurate. Thus, this contrast provides an index of task switching when the response is made based upon the correct use of the stimulus-response rule, rather than due to other possible factors. The main effect of Pure Task was significant $(F(1,135)=74.46, p<.001$, $\left.\eta_{\mathrm{p}}^{2}=.36\right)$, as was the main effect of Age $(F(1,135)=5.16, p=.03$, $\left.\eta_{\mathrm{p}}^{2}=.04\right)$. There was also a significant interaction between Pure Task and Age $\left(F(1,135)=8.097, p=.005, \eta_{\mathrm{p}}^{2}=.06\right)$. Younger adults performed worse on dual-task incongruent relative to single-task incongruent trials $(t(61)=4.63, p<.001,95 \% \mathrm{CI}[.03-.07])$, as did older adults $(t(74)=7.67, p<.001,95 \% \mathrm{CI}[.07-.12])$. However, as was reported above, whereas older and younger adults did not

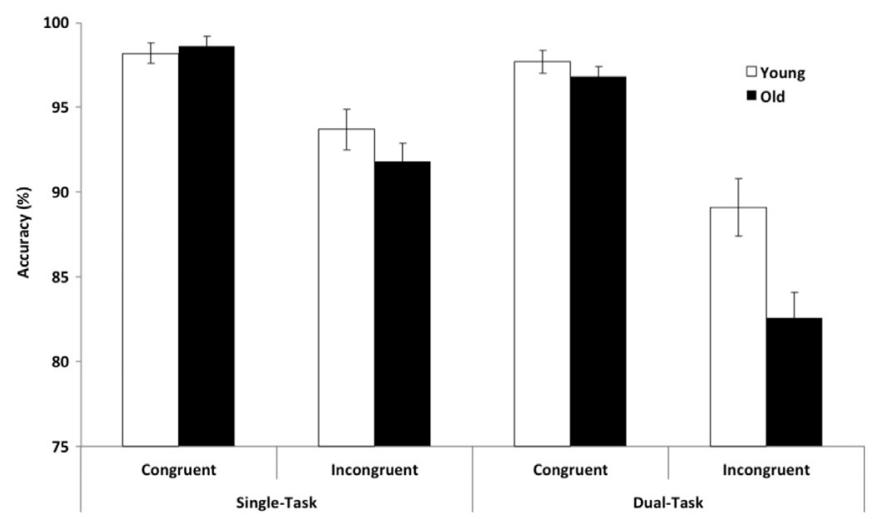

Fig. 2. Behavioral performance (percent correct) as a function of Task (no-switch, single-task trials vs. switch, dual-task trials), Congruency (congruent, where the letter stimulus requires the same stimulus-response regardless of its color vs. incongruent, where the letter stimulus calls for opposing responses depending on its color) and Age (younger and older adults). Error bars indicate SEM. Significant main effects and 2-ways interactions are moderated by a significant 3-way interaction. Posthoc analyses reveal that this interaction stems from a difference in performance between younger and older adults in the dual-task incongruent condition.

differ in performance on single-task incongruent trials $(\mathrm{t}(135)=$ $1.13, \mathrm{p}>.25$ ), older adults performed significantly worse relative to younger adults on dual-task incongruent trials $(t(135)=2.82$, $p=.006,95 \%$ CI [.02-.019]).

For the second contrast, we compared performance on singletask congruent versus single-task incongruent trials, which provides a measure of Pure Congruency (as there is no additional task load/switching present on these trials). The main effect of Pure Congruency was significant $\left(F(1,135)=54.05, p<.001, \eta_{p}^{2}=.29\right)$, but the main effect of Age was not $(F(1,135)<1)$. However, there we found a trend towards a significant interaction between Pure Congruency and Age $\left(F(1,135)=2.28, p=.13, \eta_{\mathrm{p}}^{2}=.02\right)$. Both younger and older adults had lower accuracy on single-task incongruent compared to single-task congruent trials (young: $t$ $(61)=5.21, p<.001,95 \%$ CI [.03-.06]; old: $t(74)=5.67, p<.001,95 \%$ CI [.04-.09]).

Finally, we investigate Reaction Time (RT) differences. A Task $\times$ Congruency $\times$ Age ANOVA revealed significant main effects of Task $\left(F(1,135)=930.5, p<.001, \eta_{\mathrm{p}}^{2}=.87\right)$, Congruency $(F(1,135)=$ 169.49, $\left.p<.001, \quad \eta_{\mathrm{p}}^{2}=.56\right)$ and Age $(F(1,135)=46.52, p<.001$, $\left.\eta_{\mathrm{p}}^{2}=.26\right)$ in the anticipated directions, with RTs being longer for dual compared to single-task, for incongruent relative to congruent trials, and for older relative to younger adults. The interaction between Task $\times$ Age was significant $(F(1,135)=31.15$, $\left.p<.001, \eta_{p}^{2}=.19\right)$. However, the interactions between Congruency $\times$ Age, Task $\times$ Congruency, and Task $\times$ Congruency $\times$ Age were not significant $(F \mathrm{~S}<1)$.

\section{2. fMRI data}

As is described in the methods section, for all contrasts, we first computed whole brain activation effects for younger and older adults compared to baseline, and then directly contrasted whole brain activation differences between the younger and older adults. Two sets of contrasts were performed. The first set investigated the brain-bases of switch costs. Contrast 1 was all dual-task trials greater than all single-task trial activations, and contrast 2 was dual-task incongruent trials greater than single-task incongruent trials. The second set of contrasts focused on the neural responses associated with congruency effects. Contrast 3 was all incongruent trial activations greater than all congruent trial activations, and contrast 4 was single-task incongruent trials greater than single- 
task congruent trials.

\subsubsection{Neural networks associated with task switching}

We began by comparing dual-task $>$ single-task trials, collapsing across congruency type. In the young adult group, we found two large clusters of activation. The first peaked in the left Dorsal Posterior Parietal Cortex/Precuneus (BA 7) and the second in the left Cingulate Gyrus (BA 32; Fig. 3A; File1.nii here). For the same dual-task > single-task contrast, the older adults revealed a similar pattern of activation, with clusters peaking in the left lateralized Superior Parietal Lobe (BA 7 and 19) as well as in the Cingulate Gyrus (Fig. 3B; File2.nii here). Activation was increased for the young relative to the old ${ }^{1}$ in several frontal and Occipital areas (Fig. 3C; File3.nii here). In a direct comparison between agegroups, we found numerous regions including the Middle Frontal Gyrus (MFG) and Inferior Frontal Gyrus (IFG), the ACC, and the Inferior Parietal Lobule (IPL) that were more strongly activated for the older relative to the younger adults ${ }^{1}$ (Fig. 3D; File4.nii here). Table 1 displays MNI coordinates of closest grey matter to peak cluster activations and associated anatomical label.

\subsubsection{Modulation of task switching networks by congruency}

We next reran the dual-task > single-task contrast (see Section 3.2.1), but limited to incongruent trials (dual-task incongruent $>$ single-task incongruent). This contrast provides a pure and response-conflict unbiased measure of global switch costs because it is limited to incongruent trials, and thus reflects activation differences when the participant actively, and correctly, switched between the two tasks. In other words, any effect of response-conflict congruency is factored out in this contrast. The young participants (Fig. 4A; File5.nii here) showed a similar pattern of results to the contrast of dual-task $>$ single-task, with increased activations peaking in the Precuneus (BA 7). In addition, clusters of activity peaking in a left frontal region of the MFG (BA 6 ), as well as in the Thalamus were revealed. For the older adults, activation centered around BA 8, in the Superior Frontal Gyrus (Fig. 4B; File6.nii here). Table 2 displays MNI coordinates of closest grey matter to peak cluster activations. There were no areas that younger adults activated to a greater extent than older adults, or the inverse.

\subsubsection{Neural basis of response-conflict congruency effects}

We then performed two additional contrasts exploring response-conflict level congruency effects. We first conducted whole brain group level analyses on incongruent $>$ congruent trials (collapsing across single and dual-task blocks). We found no significant activations for either the young or old adults separately when compared to baseline, or when we compared activity in the young $>$ old. However, we did find that the older adults activated an area in the Claustrum more so than did the younger adults (Fig. 5; Table 3; File7.nii here).

\subsubsection{Age-related effects of response-conflict, dissociated from task switching}

Finally, to isolate the effects of congruency alone, without the confounding of the task load (single- versus dual-task) we contrasted activations for single-task incongruent versus single-task congruent trials. In this contrast, only one task set is active (as we are investigating activity only when a decision about one task set is required). However, behaviorally, we found a significant decrease in performance accuracy (see Section 3.1) for this comparison for both older and younger adults (with a stronger effect for older adults), suggesting that the decision-rule for the non-

\footnotetext{
${ }^{1}$ Activations with a $\mathrm{z}>2.3$ and cluster extent of $\geq 20$ contiguous voxels.
}

presented task set is active in memory even under these conditions, and may be causing stimulus level response-conflict. We found no activations for either the younger or older adults separately that were significantly different than baseline, or for the young $>$ old. However, as can be seen in Fig. 6 and Table 4 (and see File8.nii here), the older adults activated specific Parietal areas including the IPL and the Superior Parietal Lobule (SPL) bordering on Primary Sensory Cortex, as well as Temporal (Superior Temporal Gyrus; STG) and Occipital (Fusiform Gyrus) areas to a greater extent than younger adults when we contrasted single-task incongruent to single-task congruent trials.

\section{Discussion}

In the current study, we used fMRI to examine and tease apart age-related differences in the brain-bases of response-conflict congruency and switching effects within a task switching paradigm. Behaviorally, we found that incongruency moderated agerelated differences in switch costs. Neurally, we found that the brain areas associated with switch costs and congruency-related response-conflict were both overlapping and distinct, and diverged as a function of age.

Numerous behavioral and neuroimaging studies have used task switching paradigms to investigate the nature of task-related representations and cognitive control in humans. Results from these studies have revealed a robust pattern of results. Behaviorally, switching between two simple tasks results in performance decrements relative to repeating the same task (Allport et al., 1994; Rogers and Monsell, 1995). Neurally, task switching has been shown to involve activation in parietal regions as well as the anterior PFC and DLPFC (DiGirolamo et al., 2001; Dreher and Grafman, 2003), areas that are associated with maintaining goalrelated information and switching attentional focus according to both animal and human neuropsychological studies (Miller and Cohen, 2001; Petersen and Posner, 2012) and are key nodes in the dorsal attention network (Cabeza, 2008; Corbetta and Shulman, 2002; Fox et al., 2006).

Age-related changes to task switching abilities, and the neural mechanisms underlying these changes, have also been well documented. Older adults consistently show increased behavioral difficulties relative to younger adults, and these performance differences have been associated with patterns of activations that differ from those of younger adults. DiGirolamo et al. (2001), for example, reported that the difference in prefrontal activation between dual- and single-task conditions was smaller for the older adults relative to younger adults. Further, older adults were shown to activate these same areas in the single-task condition, whereas the younger adults did not, suggesting that older adults have an inefficiency or reduction in some general cognitive resource that the younger adults rely on only in the face of significantly more challenging task-loads.

In the current study, we replicated previous reports showing both behavioral and neural differences between older and younger adults in task switching. We found, consistent with our own previous findings (Eich et al., 2015), that dual-task performance was less accurate than single-task performance for both younger and older adults, but younger adults performed better than older adults, particularly on the dual-task blocks. Further, we found that stimulus-response incongruency interacted with age-related differences in switch costs behaviorally.

Neurally, we found the commonly-reported pattern of global switch cost activations in the dorsal attention network for both older and younger adults, including activation in the Cingulate Gyrus and left lateralized Parietal regions (Brass and von Cramon, 2002; Braver et al., 2003; Dove et al., 2000; Dreher and Grafman, 


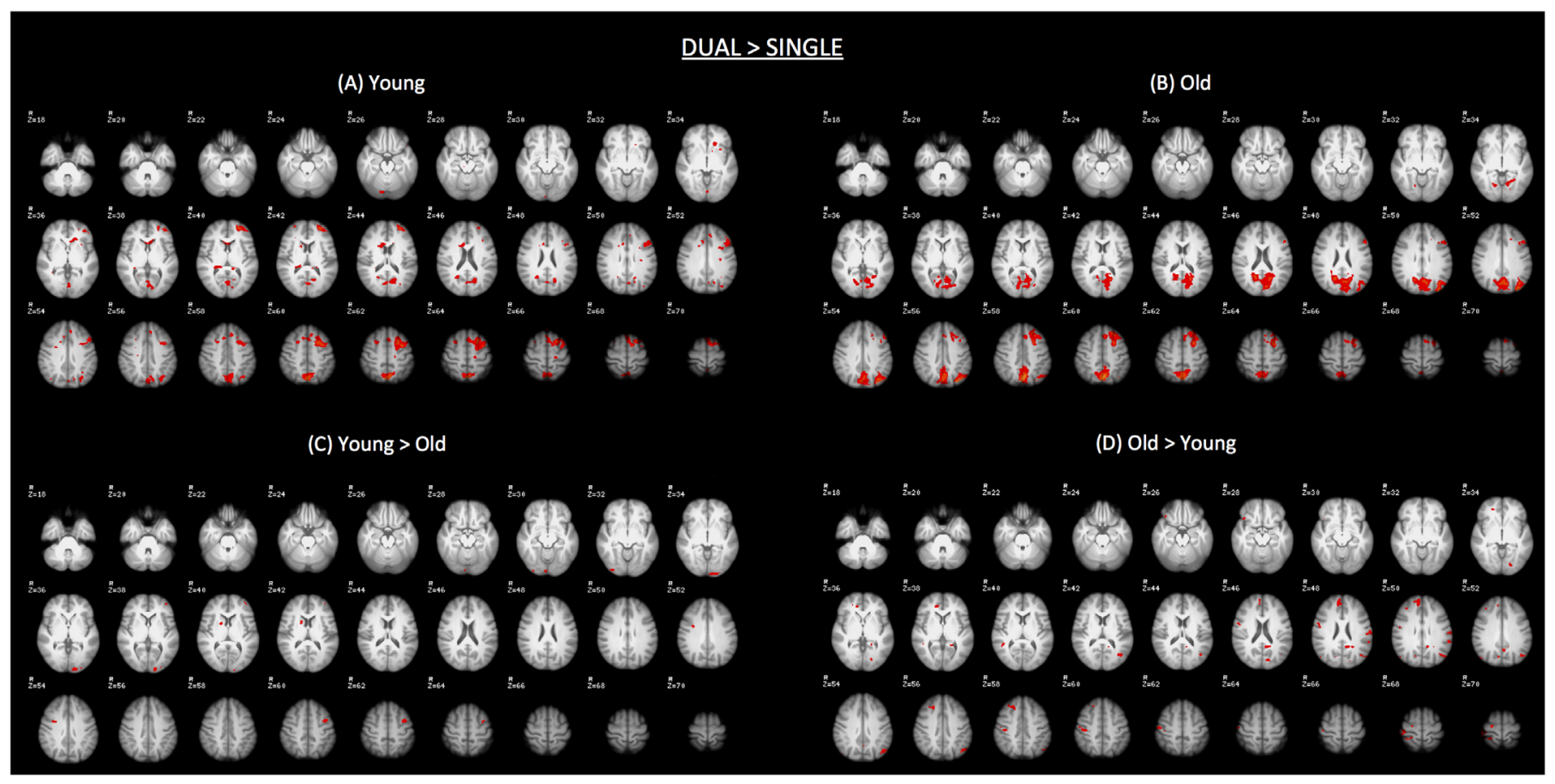

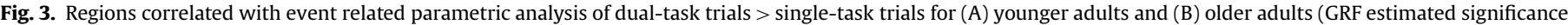

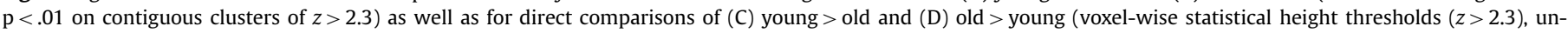
corrected, cluster extent threshold of 20 contiguous voxels).

Table 1

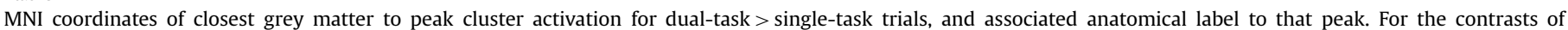

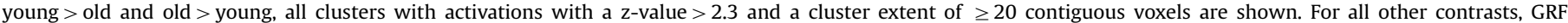
estimated significance $\mathrm{p}<.01$ on contiguous clusters of $\mathrm{z}>2.3$. $\mathrm{L}=\mathrm{Left} ; \mathrm{R}=$ Right; $\mathrm{BA}=$ Brodmann Area.

\begin{tabular}{|c|c|c|c|c|c|c|}
\hline Group & MNI coordinate $(x, y, z)$ & Hemi-sphere & Lobe & Area & $\mathbf{B A}$ & \# Voxel \\
\hline \multirow[t]{2}{*}{ Young } & $0,-70,50$ & $\mathrm{~L}$ & Parietal & Precuneus & 7 & 1581 \\
\hline & $-20,12,48$ & $\mathrm{~L}$ & Limbic & Cingulate Gyrus & 32 & 2304 \\
\hline \multirow[t]{3}{*}{ Old } & $-32,-78,40$ & $\mathrm{~L}$ & Parietal & Precuneus & 19 & 879 \\
\hline & $-20,12,48$ & $\mathrm{~L}$ & Limbic & Cingulate Gyrus & 32 & 1754 \\
\hline & $0,-72,46$ & $\mathrm{~L}$ & Parietal & Precuneus & 7 & 5408 \\
\hline \multirow[t]{7}{*}{ Young $>$ Old } & $-38,52,8$ & $\mathrm{~L}$ & Frontal & Middle Frontal Gyrus & 10 & 20 \\
\hline & $8,-92,-12$ & $\mathrm{R}$ & Occipital & Lingual Gyrus & 18 & 28 \\
\hline & $36,-90,-8$ & & & Inferior Occipital Gyrus & & 34 \\
\hline & $16,8,12$ & $\mathrm{R}$ & Sub-Lobular & Caudate & - & 38 \\
\hline & $40,0,34$ & $\mathrm{R}$ & Frontal & Precentral Gyrus & 6 & 44 \\
\hline & $-38,2,50$ & $\mathrm{~L}$ & Frontal & Middle Frontal Gyrus & 6 & 103 \\
\hline & $-10,-96,-4$ & $\mathrm{~L}$ & Occipital & Lingual Gyrus & 17 & 152 \\
\hline \multirow[t]{21}{*}{ Old $>$ Young } & $8,-74,32$ & $\mathrm{R}$ & Parietal & Precuneus & 31 & 20 \\
\hline & $36,36,28$ & $\mathrm{R}$ & Frontal & Middle Frontal Gyrus & 9 & 20 \\
\hline & $46,-78,32$ & $\mathrm{R}$ & Occipital & Superior Occipital Gyrus & 19 & 22 \\
\hline & $-16,-46,2$ & $\mathrm{~L}$ & Limbic & Parahippocampal Gyrus & 30 & 25 \\
\hline & $44,-36,10$ & $\mathrm{R}$ & Temporal & Superior Temporal Gyrus & 41 & 26 \\
\hline & $46,22,-18$ & $\mathrm{R}$ & Frontal & Inferior Frontal Gyrus & 47 & 27 \\
\hline & $-10,-82,22$ & $\mathrm{~L}$ & Occipital & Cuneus & 18 & 27 \\
\hline & $-4,-58,32$ & $\mathrm{~L}$ & Limbic & Cingulate Gyrus & 31 & 28 \\
\hline & $-18,-80,-2$ & $\mathrm{~L}$ & Occipital & Lingual Gyrus & 18 & 35 \\
\hline & $-62,-40,28$ & $\mathrm{~L}$ & Parietal & Inferior Parietal Lobule & 40 & 37 \\
\hline & $22,-12,68$ & $\mathrm{R}$ & Frontal & Precentral Gyrus & 6 & 40 \\
\hline & $54,0,20$ & & & & & 58 \\
\hline & $-42,-70,10$ & $\mathrm{~L}$ & Occipital & Middle Occipital Gyrus & 19 & 59 \\
\hline & $26,-40,66$ & $\mathrm{R}$ & Limbic & Anterior Cingulate & 32 & 70 \\
\hline & $-66,-20,26$ & $\mathrm{~L}$ & Parietal & Postcentral Gyrus & 2 & 75 \\
\hline & $-8,-56,26$ & $\mathrm{~L}$ & Limbic & Posterior Cingulate & 31 & 96 \\
\hline & $22,28,42$ & $\mathrm{R}$ & Frontal & Superior Frontal Gyrus & 8 & 99 \\
\hline & $46,-32,66$ & $\mathrm{R}$ & Parietal & Postcentral Gyrus & 2 & 103 \\
\hline & $42,-22,44$ & & & & & 130 \\
\hline & $8,54,28$ & $\mathrm{R}$ & Frontal & Superior Frontal Gyrus & 9 & 183 \\
\hline & $-44,-70,40$ & $\mathrm{~L}$ & Parietal & Precuneus & 39 & 211 \\
\hline
\end{tabular}




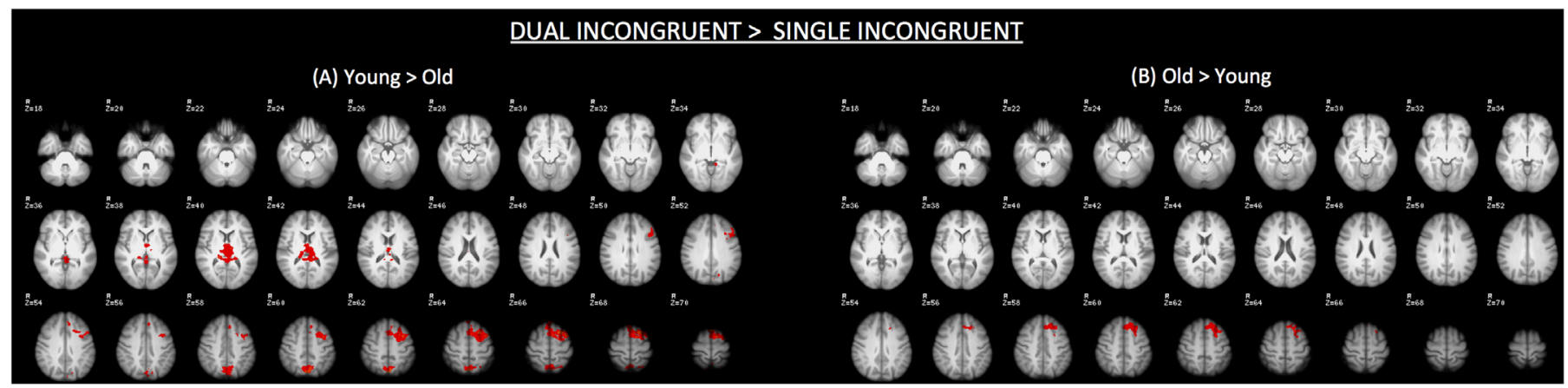

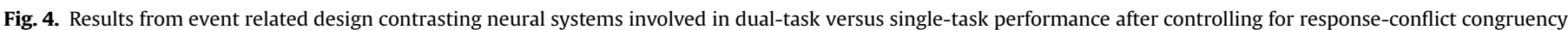

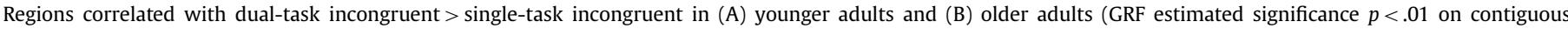
clusters of $z>2.3$ ).

Table 2

MNI coordinates of closest grey matter to peak cluster activation for dual-task incongruent $>$ single-task incongruent trials that reached statistical significance, and associated anatomical label to that peak. GRF estimated significance $\mathrm{p}<.01$ on contiguous clusters of $z>2.3$. No clusters survived thresholding for young $>$ old or old $>$ young. $\mathrm{L}=$ Left; $\mathrm{R}=$ Right; $\mathrm{BA}=$ Brodmann Area.

\begin{tabular}{|c|c|c|c|c|c|c|}
\hline Group & $\begin{array}{l}\text { MNI coordinate } \\
(x, y, z)\end{array}$ & $\begin{array}{l}\text { Hemi- } \\
\text { sphere }\end{array}$ & Lobe & Area & BA & \# Voxel \\
\hline \multirow[t]{3}{*}{ Young } & $-12,16,62$ & $\mathrm{~L}$ & Frontal & $\begin{array}{l}\text { Middle Frontal } \\
\text { Gyrus }\end{array}$ & 6 & 2739 \\
\hline & $-8,-14,10$ & $\mathrm{~L}$ & - & Thalamus & - & 1234 \\
\hline & $0,-72,54$ & $\mathrm{~L}$ & Parietal & Precuneus & 7 & 940 \\
\hline Old & $-18,28,46$ & $\mathrm{~L}$ & Frontal & $\begin{array}{l}\text { Superior Fron- } \\
\text { tal Gyrus }\end{array}$ & 8 & 918 \\
\hline
\end{tabular}

2003; MacDonald et al., 2000; Sohn et al., 2000). Specifically, we saw greater activations in BA 32, bordering on Area 6, when we compared dual-task trials to single-task trials (see Section 3.2.1). Consistent with previous literature, we also found on direct comparison between the old and younger adults, that older adults activated numerous additional areas, including right lateralized Frontal regions as well as bilateral areas in the dorsal attention network to a greater extent than did the younger adults. In addition, the older adults showed increased activations in the IPL, which is associated with the ventral attention network (Cabeza, 2008; Corbetta and Shulman, 2002; Fox et al., 2006) relative to
Table 3

MNI coordinates of closest grey matter to peak cluster activation for incongruent $>$ congruent that reached statistical significance, and associated anatomical label to that peak. GRF estimated significance $\mathrm{p}<.01$ on contiguous clusters of $z>2.3$. No clusters survived thresholding for young, old, or young $>$ old $\mathrm{L}=$ Left; $\mathrm{R}=$ Right; $\mathrm{BA}=$ Brodmann Area.

\begin{tabular}{llllll}
\hline Group & $\begin{array}{l}\text { MNI Coordinate } \\
(\mathbf{x}, \mathbf{y}, \mathbf{z})\end{array}$ & $\begin{array}{l}\text { Hemi- } \\
\text { sphere }\end{array}$ & Lobe & Area & BA \# Voxel \\
\hline Old $>$ Young & $-28,8,8$ & L & - & Claustrum - & 1101 \\
\hline
\end{tabular}

younger adults. These different activations however, both in our own paradigm and in the majority of task switching paradigms that use bivalent stimuli to study the nature of task-related representations and cognitive control (Monsell, 2003), may confound multiple cognitive control processes occurring during task switching. These include processes responsible for keeping more than one task set active in working memory, processes that are needed to determine which task set to perform next (see Kray and Lindenberger (2000)), as well as processes involved in determining and acting upon the appropriate stimulus-response decision rule for that trial, or those that might be influenced by the congruency of the stimulus. Thus, we attempted to factor out the effect of differential congruency to obtain a measure of pure task switching by comparing dual-task incongruent trials to single-task incongruent trials (Section 3.2.2). When we did this, we no longer

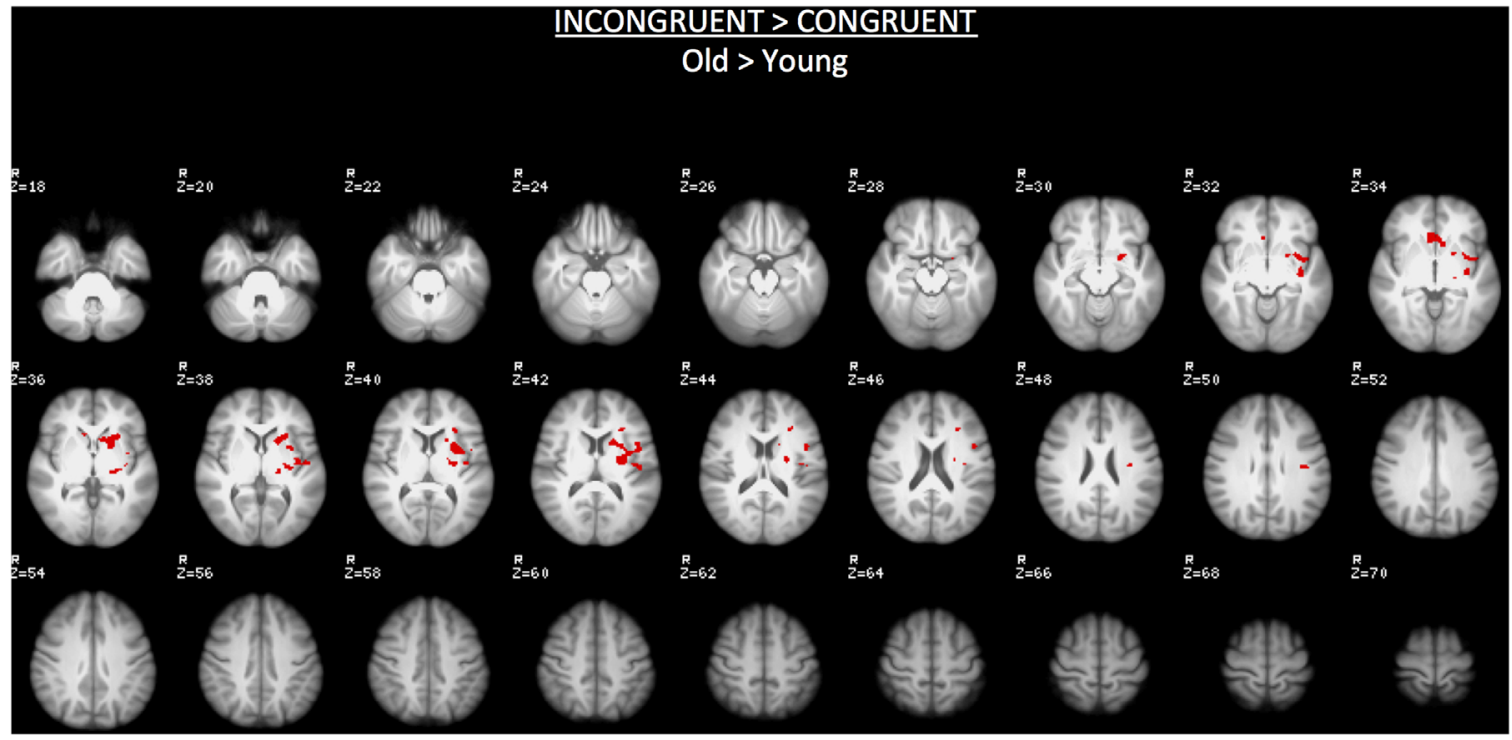

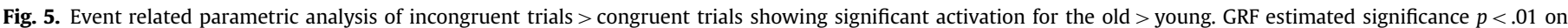
contiguous clusters of $z>2.3$. 


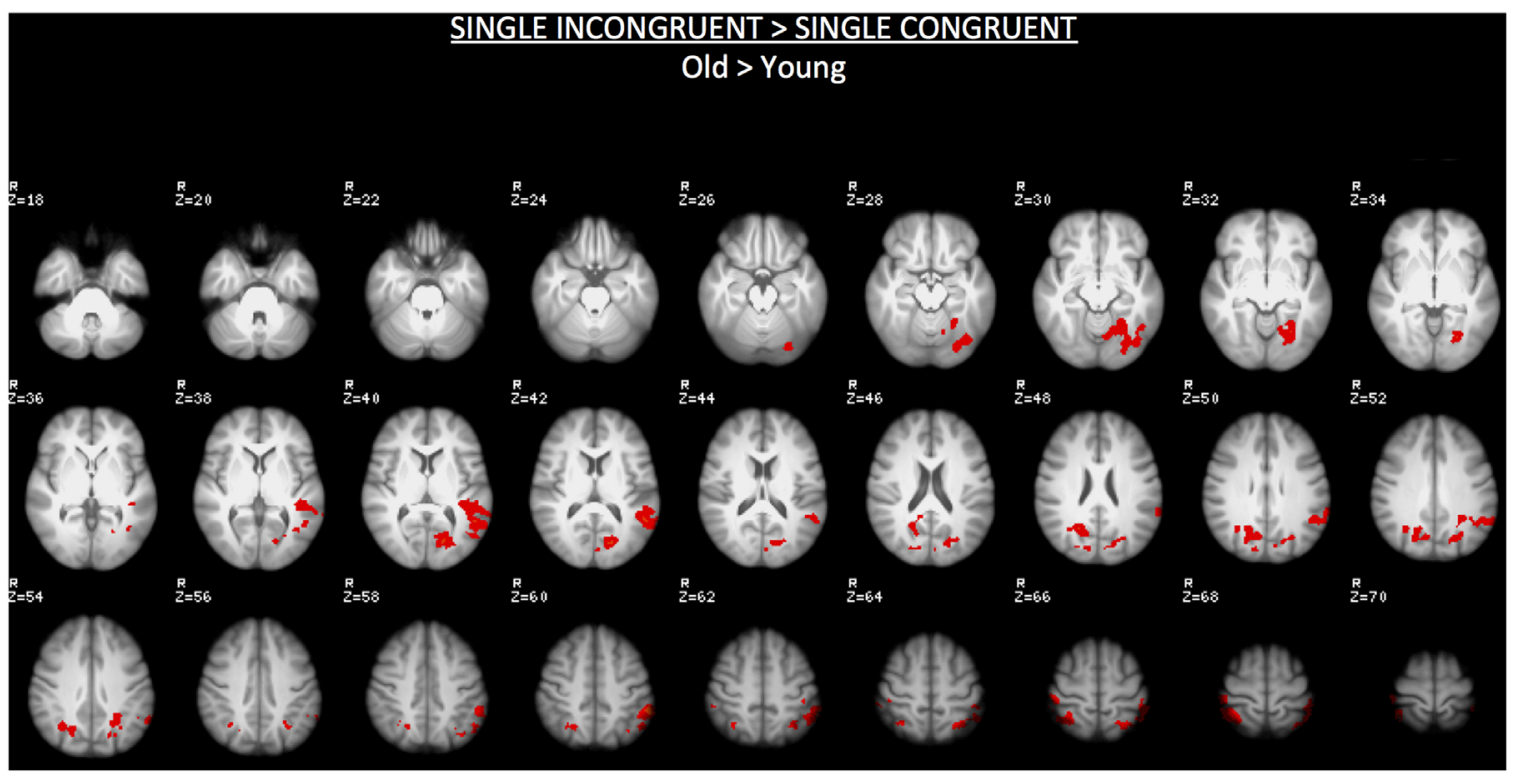

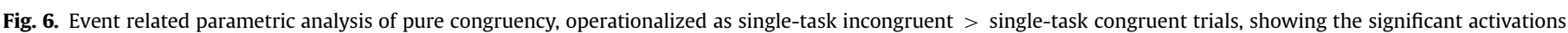

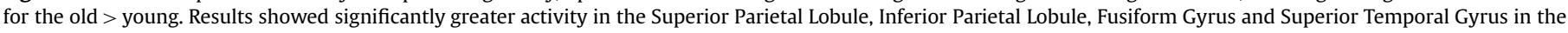
older adults compared to the younger adults. GRF estimated significance $p<.01$ on contiguous clusters of $z>2.3$.

found IPL activations for older relative to younger adults, suggesting that the IPL activations present in the dual-task $>$ singletask comparison may have been driven by the incongruent trials nested within this contrast. We did, however, find that for the older adults, switching-related activation shifted from being caudally located along the lateral axis of the PFC to being more rostrally located, in BA 8. Koechlin et al. (2003) and Koechlin and Summerfield (2007) reported a caudal-rostral lateral PFC shift as tasks requiring cognitive control increased in temporal proximity and complexity: rostral areas of the PFC were involved in more temporally distant operations, while more caudally located PFC activations were involved in more temporally proximate operations. For the contrast of dual-task incongruent trials $>$ single-task incongruent trials, what has been factored out is the stimulusresponse action, which presumably is the last and most proximate operation when making a decision about a stimulus in a task switching paradigm using bivalent stimuli.

However, our primary aim was to investigate the role of response-conflict congruency within the context of task switching. Congruency is common to many task switching paradigms, yet its impact upon switch costs goes frequently unreported, and has not yet been explored neurally in this context. To investigate the role of congruency, we first compared incongruent to congruent trials, collapsing across single- and dual-tasks (see Section 3.2.3). We found only one significant cluster of activation, for older adults relative to younger adults, which revealed greater activity peaking in the Claustrum. Although relatively little is known about the function of the Claustrum, one prominent theory posits that it is involved in "cross modal" processing, or the integration of diverse attributes of a modal perceptions (Crick and Koch, 2005).
To investigate the role of sensorimotor congruency without the confound of task switching, we then compared activation for single incongruent $>$ single congruent trials (Section 3.2.4). As was described earlier, because bivalent stimuli were used and participants both underwent a lengthy experimental training regimen and completed many experimental blocks, potent associations between stimuli and responses were likely to be present, even in single-task blocks. Indeed, behaviorally, both the older and younger adults performed worse on the single-task incongruent relative to single-task congruent trials. However, the interaction between Congruency and Age, which bordered on significance, suggests that resolving incongruency between response options might be more difficult for the older adults. In the imaging data, we found that the older adults activated areas in the dorsal attention network that were also activated when we examined switch costs associated with being in the dual-task versus the single-task condition of the paradigm, including the SPL (BA 7) and Fusiform gyrus (BA 19). In addition, we found activations in the left IPL (BA 40). The IPL is not a homogeneous region, and has been associated with numerous cognitive functions including executive control (Uddin et al., 2011), salience detection (Seeley et al., 2007) and sensory-motor operations (Iacoboni, 2005; Keysers and Gazzola, 2009). According to Ptak (2012), the IPL is "preferentially activated when a stimulus of high behavioral relevance (e.g., a stimulus that possesses some target-defining properties) appears at an unexpected position" (Corbetta et al., 2000; Indovina and Macaluso, 2007; Serences et al., 2005) suggesting that this region is important for the interruption of current cognitive activity and the reorienting of attention (Corbetta et al., 2008). Indeed, the younger adults deactivated these areas, whereas the older adults

Table 4

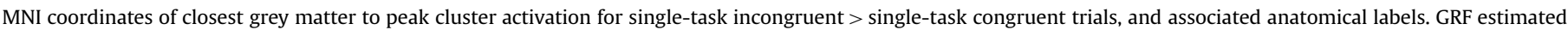
significance $\mathrm{p}<.01$ on contiguous clusters of $\mathrm{z}>2.3$. No clusters survived thresholding for young, old, or young $>$ old. $\mathrm{L}=\mathrm{Left}$; $\mathrm{R}=\mathrm{Right}$; $\mathrm{BA}=\mathrm{Brodmann}$ Area.

\begin{tabular}{|c|c|c|c|c|c|c|}
\hline Group & MNI coordinate $(x, y, z)$ & Hemi-sphere & Lobe & Area & BA & \# Voxel \\
\hline \multirow{5}{*}{ Old $>$ Young } & $38,-46,66$ & $\mathrm{R}$ & Parietal & Superior Parietal Lobule & 7 & 1095 \\
\hline & $-58,-42,48$ & $\mathrm{~L}$ & Parietal & Inferior Parietal Lobule & 40 & 1081 \\
\hline & $-22,-68,-6$ & $\mathrm{~L}$ & Occipital & Fusiform & 19 & 799 \\
\hline & $-54,-52,12$ & $\mathrm{~L}$ & Temporal & Superior Temporal Gyrus & 22 & 772 \\
\hline & $-16,-74,14$ & $\mathrm{~L}$ & Occipital & - & 17 & 678 \\
\hline
\end{tabular}


recruited them to complete the task under these conditions.

In conclusion, we found that alternating between completing two simple tasks versus completing either task alone produced reliable behavioral and neural differences in both younger and older adults. We also found specific age-related differences in these processes, primarily when the cognitive component of response-conflict congruency was taken into account. Behaviorally, older adults' performance was particularly affected on dual-task incongruent trials, which suggest that the typically seen age-related increases in switch costs may be derivative upon a more basic response-conflict congruency effect. Neurally, we found that older adults recruit areas in both the dorsal and ventral attention networks, including the STG and distinct Parietal areas, to overcome response-conflict - even in single-task blocks - to a greater extent than did younger adults. The activation of these additional brain areas, coupled with the behavioral data results, suggests that older adults may need to up-regulate the dorsal attention network and additionally recruit the ventral network in a compensatory manner to cope with the increased executive sensorimotor demand that incongruency fosters. Together, our finding indicate that congruency plays a major behavioral role in older adults' ability to switch between tasks, and also points to the fact that the brain correlates of task switching and resolving response-conflict inherent within task switching paradigms may rely on separable neural mechanisms.

\section{Acknowledgments}

Support for this publication was provided by the U.S. Department of Health and Human Services-National Institutes of HealthNational Institute of Mental Health (T32 MH020004) to Teal S. Eich and the U.S. Department of Health and Human Services-National Institutes of Health-National Institute on Aging (R01 AG-026158) to Yaakov Stern.

\section{Appendix A. Supporting information}

Supplementary data associated with this article can be found in the online version at http:dx.doi.org/10.1016/j.neuropsychologia. 2016.08.009.

\section{References}

Allport, A., Styles, E., Hsieh, S., 1994. Shifting intentional set: exploring the dynamic control of tasks. In: Umilta, C., Moscovitch, M. (Eds.), Attention and Performance XV: Conscious and Nonconscious Information Processing. MIT Press, Cambridge, MA, pp. 421-452.

Badre, D., Wagner, A.D., 2006. Computational and neurobiological mechanisms underlying cognitive flexibility. Proc. Natl. Acad. Sci. USA 103 (18), 7186-7191.

Botvinick, M., Braver, T., Barch, D., Carter, C., Cohen, J., 2001. Conflict monitoring and cognitive control. Psychol. Rev. 108 (3), 624-652.

Botwinick, J., Brinley, J.F., Robbin, J.S., 1958. Task alternation time in relation to problem difficulty and age. J. Gerontol. 13 (4), 414-417.

Brass, M., von Cramon, D., 2002. The role of the frontal cortex in task preparation. Cereb. Cortex 12 (9), 908-914.

Braver, T., Reynolds, J., Donaldson, D., 2003. Neural mechanisms of transient and sustained cognitive control during task switching. Neuron 39, 713-726.

Cabeza, R., 2002. Hemispheric asymmetry reduction in older adults: the HAROLD model. Psychol. Aging 17, 85-100.

Cabeza, R., 2008. Role of parietal regions in episodic memory retrieval: the dual attentional processes hypothesis. Neuropsychologia 46, 1813-1827.

Cabeza, R., Anderson, N.D., Locantore, J.K., McIntosh, A.R., 2002. Aging gracefully compensatory brain activity in high-performing older adults. Neuroimage 17 (3), 1394-1402.

Corbetta, M., Kincade, J., Ollinger, J., McAvoy, M., Shulman, G., 2000. Voluntary orienting is dissociated from target detection in human posterior parietal cortex. Nat. Neurosci. 3, 292-297.

Corbetta, M., Patel, G., Shulman, G., 2008. The reorienting system of the human brain: from environment to theory of mind. Neuron 58, 306-324.

Corbetta, M., Shulman, G., 2002. Control of goal-directed and stimulus-driven attention in the brain. Nat. Rev. Neurosci. 3, 215-229.

Crick, F.C., Koch, C., 2005. What is the function of the claustrum? Philos. Trans. R. Soc. Lond. B: Biol. Sci. 360 (1458), 1271-1279.

Derrfuss, J., Brass, M., Neumann, J., von Cramon, D., 2005. Involvement of the inferior frontal junction in cognitive control: meta-analyses of switching and Stroop studies. Hum. Brain Mapp. 25 (1), 22-34.

Derrfuss, J., Brass, M., von Cramon, D., 2004. Cognitive control in the posterior frontolateral cortex: evidence from common activations in task coordination, interference control, and working memory. Neuroimage 23 (2), 604-612.

DiGirolamo, G.J., Kramer, A.F., Barad, V., Cepeda, N.J., Weissman, D.H., Milham, M.P. McAuley, E., 2001. General and task-specific frontal lobe recruitment in older adults during executive processes: a fMRI investigation of task switching. Neuroreport 12 (9), 2065-2071.

Dove, A., Pollman, S., Schubert, T., Wiggins, C., von Cramon, D., 2000. Prefrontal cortex activation in task switching: an event-related fMRI study. Cogn. Brain Res. 9, 103-109.

Dreher, J., Grafman, J., 2003. Dissociating the roles of the rostral anterior cingulate and the lateral prefrontal cortices in performing two tasks simultaneously or successively. Cereb. Cortex 13 (4), 329-339.

Durston, S., Davidson, M., Thomas, K., Worden, M., Tottenham, N., Martinez, A., Casey, B., 2003. Parametric manipulation of conflict and response competition using rapid mixed-trial event-related fMRI. Neuroimage 20 (4), 2135-2141.

Eich, T.S., Rakitin, B.C., Stern, Y., 2015. Response-conflict moderates the cognitive control of episodic and contextual load in older adults. J. Gerontol.: Psychol. Sci.

Eriksen, B.A., Eriksen, C.W., 1974. Effects of noise letters upon identification of a target letter in a non-search task. Percept. Psychophys. 16, 143-149.

Fagot, C., 1994. Chronometric investigations of task switching. Dissertation.

Fan, J., Flombaum, J.I., McCandliss, B.D., Thomas, K.M., Posner, M.I., 2003. Cognitive and brain consequences of conflict. Neuroimage 18 (1), 42-57.

Fitts, P.M., Deininger, R.L., 1954. S-R compatibility: correspondence among paired elements within stimulus and response codes. J. Exp. Psychol. 48 (6), 483-492.

Fitts, P.M., Seeger, C.M., 1953. S-R compatibility: spatial characteristics of stimulus and response codes. J. Exp. Psychol. 46 (3), 199-210.

Fox, M.D., Corbetta, M., Snyder, A.Z., Vincent, J.L., Raichle, M.E., 2006. Spontaneous neuronal activity distinguishes human dorsal and ventral attention systems. Proc. Natl. Acad. Sci. USA 103 (26), 10046-10051.

Gopher, D., Armony, L., Greenshpan, Y., 2000. Switching tasks and attention policies. J. Exp. Psychol.: Gen. 129, 308-339.

Hartley, A.A., Kieley, J.M., Slabach, E.H., 1990. Age Differences and similarities in the effects of cues and prompts. J. Exp. Psychol.: Hum. Percept. Perform. 16, 523-537.

Iacoboni, M., 2005. Neural mechanisms of imitation. Curr. Opin. Neurobiol. 15 (6), 632-637.

Indovina, I., Macaluso, E., 2007. Dissociation of stimulus relevance and saliency factors during shifts of visuospatial attention. Cereb. Cortex 17, 1701-1711.

Jersild, A.T., 1927. Mental Set and Shift (Issued Also as Thesis1927 Columbia Univ). New York.

Kane, M.J., Engle, R.W., 2003. Working-memory capacity and the control of attention: The contributions of goal maintenance, response competition, and task set to Stroop interference. J. Exp. Psychol.: Gen. 132, 47-70.

Keysers, C., Gazzola, V., 2009. Expanding the mirror: vicarious activity for actions emotions, and sensations. Curr. Opin. Neurobiol. 19 (6), 666-671.

Kiesel, A., Steinhauser, M., Wendt, M., Falkenstein, M., Jost, K., Philipp, A., Koch, I., 2010. Control and interference in task switching-A review. Psychol. Bull. 136 (5), 849.

Koechlin, E., Ody, C., Kouneiher, F., 2003. The architecture of cognitive control in the human prefrontal cortex. Science 302 (5648), 1181-1188.

Koechlin, E., Summerfield, C., 2007. An information theoretical approach to prefrontal executive function. Trends Cogn. Sci. 11 (6), 229-235.

Kornblum, S., Lee, J.-W., 1995. Stimulus-response compatibility with relevant and irrelevant stimulus dimensions that do and do not overlap with the response. J. Exp. Psychol.: Hum. Percept. Perform. 21 (4), 855-875.

Kramer, A.F., Hahn, S., Gopher, D., 1999. Task coordination and aging: explorations of executive control processes in the task switching paradigm. Acta Psychol. 101 (2-3), 339-378.

Kray, J., Lindenberger, U., 2000. Adult age differences in task switching. Psychol. Aging 15, 126-147.

Lien, M.-C., Proctor, R.W., 2002. Stimulus-response compatibility and psychological refractory period effects: implications for response selection. Psychon. Bull. Rev. 9 (2), 212-238.

MacDonald, A., Cohen, J., Stenger, V., Carter, C., 2000. Dissociating the role of dorsolateral prefrontal and anterior cingulate cortex in cognitive control. Science 288, 1835-1838.

MacLeod, C.M., 1991. Half a century of research on the Stroop effect: an integrative review. Psychol. Bull. 109, 163-203.

Macwhinney, B., Cohen, J., Provost, J., 1997. The PsyScope experiment-building system. Spat. Vis. 11 (1), 99-101.

Mattis, S., 1988. Dementia Rating Scale, Professional Manual. Psychological Assessment Resources Inc., Odessa, FL.

Mayr, U., 2001. Age differences in the selection of mental sets: the role of inhibition, stimulus ambiguity, and response-set overlap. Psychol. Aging 16, 96-109.

Meiran, N., 2000a. Modeling cognitive control in task switching. Psychol. Res. 63, 234-249.

Meiran, N., 2000b. Reconfiguration of stimulus task sets and response task sets 
during task switching. In: Monsell, S., Driver, J. (Eds.), Control of Cognitive Processes: Attention and Performance XVIII. MIT Press, Cambridge, MA, pp. 377-400.

Meiran, N., 2005. Task rule-congruency and Simon-like effects in switching between spatial tasks. Q. J. Exp. Psychol. A 58, 1023-1041.

Meiran, N., Gotler, A., Perlman, A., 2001. Old is associated with a pattern of relatively impaired and relatively intact task set switching abilities. J. Gerontol.: Psychol. Sci. 56B, 88-102.

Meiran, N., Kessler, Y., 2008. The task rule congruency effect in task switching reflects activated long-term. J. Exp. Psychol.: Hum. Percept. Perform. 34, 137-157.

Milham, M., Banich, M., 2005. Anterior cingulate cortex: an fMRI analysis of conflict specificity and functional differentiation. Hum. Brain Mapp. 25 (3), 328-335.

Milham, M., Banich, M., Barad, V., 2003. Competition for priority in processing increases prefrontal cortex's involvement in top-down control: an event-related fMRI study of the Stroop Task. Cogn. Brain Res. 17, 212-222.

Milham, M., Erickson, K., Banich, M., Kramer, A., Webb, A., Wszalek, T., Cohen, N., 2002. Attentional control in the aging brain: insights from an fMRI study of the Stroop task. Brain Cogn. 49, 277-296.

Miller, E., Cohen, J., 2001. An integrative theory of prefrontal cortex function. Annu. Rev. Neurosci. 24, 167-202.

Monsell, S., 2003. Task switching. Trends Cogn. Sci. 7 (3), 134-140.

Monsell, S., Sumner, P., Waters, H., 2003. Task set reconfiguration with predictable and unpredictable task switches. Mem. Cogn. 31 (3), 327-342.

Pashler, H., 1994. Dual-task interference in simple tasks: data and theory. Psychol. Bull. 116 (2), 220-244. http://dx.doi.org/10.1037/0033-2909.116.2.220.

Petersen, S.E., Posner, M.I., 2012. The attention system of the human brain: 20 years after. Annu. Rev. Neurosci. 35, 73-89.

Ptak, R., 2012. The Frontoparietal Attention Network of the Human Brain: Action, Saliency, and a Priority Map of the Environment. Neuroscientist. 18 (5), 502-515.

Reynolds, J., O'Reilly, R., 2009. Developing PFC representations using reinforcement learning. Cognition 113 (3), 281-292.

Rogers, R.D., Monsell, S., 1995. The costs of a predictable switch between simple cognitive tasks. J. Exp. Psychol.: Gen. 124, 207-231.
Seeley, W., Menon, V., Schatzberg, A., Keller, J., Glover, G., Kenna, H., Greicius, M. 2007. Dissociable intrinsic connectivity networks for salience processing and executive control. J. Neurosci. 27 (9), 2349-2356.

Serences, J., Shomstein, S., Leber, A., Golay, X., Egeth, H., Yantis, S., 2005. Coordination of voluntary and stimulus-driven attentional control in human cortex. Psychol. Sci. 16 (114-22).

Simon, J.R., Rudell, A.P., 1967. Auditory S-R compatibility: the effect of an irrelevant cue on information processing. J. Appl. Psychol. 51, 300-304.

Sohn, M.-H., Ursu, S., Anderson, J., Stenger, V., Carter, C., 2000. The role of prefrontal cortex and posterior parietal cortex in task switching. Proc. Natl. Acad. Sci. USA 97, 13448-13453.

Stroop, J.R., 1935. Studies of interference in serial verbal reactions. J. Exp. Psychol. 18 (6), 643-662.

Sudevan, P., Taylor, D., 1987. The cuing and priming of cognitive operations. J. Exp, Psychol.: Hum. Percept. Perform. 13 (1), 89-103.

Uddin, L., Supekar, K., Ryali, S., Menon, V., 2011. Dynamic reconfiguration of structural and functional connectivity across core neurocognitive brain networks with development. J. Neurosci. 31 (50), 18578-18589.

Ullsperger, M., von Cramon, D., 2003. Error monitoring using external feedback: specific roles of the habenular complex, the reward system, and the cingulate motor area revealed by functional magnetic resonance imaging. J. Neurosci. 23 (10), 4308-4314.

vanVeen, V., Carter, C., 2005. Separating semantic conflict and response-conflict in the Stroop task: a functional MRI study. Neuroimage 27, 497-504.

Verhaeghen, P., 2011. Aging and executive control: reports of a demise greatly exaggerated. Curr. Dir. Psychol. Sci. 20 (3), 174-180.

Verhaeghen, P., Cerella, J., 2002. Aging, executive control, and attention: a review of meta-analyses. Neurosci. Biobehav. Rev. 26 (7), 849-857.

Wasylyshyn, C., Verhaeghen, P., Sliwinski, M., 2011. Aging and task switching: a meta-analysis. Psychol. Aging 26 (1), 15-20.

Worsley, K.J., Evans, A.C., Marrett, S., Neelin, P., 1992. A three-dimensional statistical analysis for CBF activation studies in human brain. J. Cereb. Blood Flow. Metab. 12, 900-918. 